\title{
Electron Transport at Surfaces and Interfaces
}

\author{
Christian A. Bobisch* and Rolf Möller*
}

\begin{abstract}
Here we present two techniques which give insight on transport phenomena with atomic resolution. Ballistic electron emission microscopy is used to study the ballistic transport through layered heterogeneous systems. The measured ballistic fraction of the tunneling current provides information about lossless transport channels through metallic layers and organic adsorbates. The transport characteristics of $\mathrm{Bi}(111) / \mathrm{Si} \mathrm{Schottky}$ devices and the influence of the organic adsorbates perylene tetracaboxylic dianhydride acid and $\mathrm{C}_{60}$ on the ballistic current are discussed. Scanning tunneling potentiometry gives access to the lateral transport along a surface, thus scattering processes within two-dimensional electron systems for the $\mathrm{Bi}(111)$ surface and the $\mathrm{Si}(111)(\sqrt{ } 3 \times \sqrt{ } 3)-$ Ag surface could be visualized.
\end{abstract}

Keywords: Ballistic electron emission microscopy $\cdot \mathrm{Bi}(111) \cdot \mathrm{C}_{60} \cdot$ Electron transport $\cdot \mathrm{PTCDA} \cdot$ Scanning tunneling potentiometry

\section{Introduction}

Three decades after its invention, the scanning tunneling microscope (STM) ${ }^{[1,2]}$ is a standard tool to characterize surface phenomena on the atomic scale. The STM enables surface morphology to be imaged with atomic resolution on metals, semiconductors or thin insulating layers. ${ }^{[3-7]}$ In addition, organic adsorbates such as single molecules or self-ordered layers can be studied. ${ }^{[8,9]}$ Moreover, spectroscopic measurements allow the characterization of the electronic structure of surfaces ${ }^{[10,11]}$ and adsorbed molecules, for example. Since the derivative of the tunneling current versus the tunneling bias carries information about the local density of states as a function of energy, molecular orbitals of adsorbates, for example, can be studied with the high lateral resolution of the STM. ${ }^{[12]}$

Different STM-based methods are available which allow the study of, e.g. vibronic properties, ${ }^{[13,14]}$ magnetic properties ${ }^{[15,16]}$ or tunneling current-induced luminescence ${ }^{[17-19]}$ down to the atomic scale.

\footnotetext{
${ }^{\star}$ Correspondence: Dr. C. A. Bobisch, Prof. Dr. R. Möller

University of Duisburg-Essen

Faculty of Physics and CeNIDE - Center for Nanointegration Duisburg-Essen

Lotharstr. 1

D-47048 Duisburg, Germany

Tel.: +492033794220

Fax: +49203379 1727

E-mail: christian.bobisch@uni-due.de,

rolf.moeller@uni-due.de
}

All these techniques rely on a conventional two-terminal approach to conduct scanning tunneling microscopy.

Here we want to discuss two extended STM-based methods, ballistic electron emission microscopy $(\mathrm{BEEM})^{[20,21]}$ and scanning tunneling potentiometry (STP) ${ }^{[22,23]}$ which are three terminal extensions to the conventional STM. Both of them allow the study of transport phenomena with atomic resolution. BEEM measures transport orthogonal to the surface or an interface. Ballistic transport across the $\mathrm{Bi}(111) / \mathrm{Si}$ Schottky interface will be discussed. ${ }^{[24,25]}$ Using an alternative BEEM geometry, the ballistic transport through two archetype organic molecules (the fullerene $\mathrm{C}_{60}$ and the perylene derivate perylene-3,4,9,10-tetracarboxylic dianhydride (PTCDA)) adsorbed onto the $\mathrm{Bi}(111)$ surface is studied. Both molecules are extensively studied by scanning tunneling microscopy and spectroscopy. ${ }^{[26-28]}$ In contrast to established BEEM techniques which rely on placing the organic material at the Schottky interface between the metal layer and the base semiconductor, ${ }^{[29,30]}$ we choose a geometry where the organic molecules are deposited on top of the $\mathrm{Bi}(111)$ layer. This preserves the high quality of the metal film and the interface and allows transport through molecules with submolecular resolution to be studied.[31,32]

Charge transport parallel to a surface or an interface is studied by STP measuring the variation of the electrochemical potential, while a lateral current is applied through the surface structure. We apply this techniques to study transport phenomena at the surface of $\mathrm{Bi}(111)$ and the silverinduced reconstructed $\operatorname{Si}(111)(\sqrt{3} \times \sqrt{3})-\mathrm{Ag}$ surface, i.e. electron transport within the two-dimensional electron gas of the surface state.

\section{Experiment and Sample Preparation}

All measurements were carried out at ultra high vacuum conditions $\left(\mathrm{p} \sim 10^{-10}\right.$ mbar) in a four probe STM (Nanoprobe by Omicron) which was modified in order to obtain atomic resolution imaging. The microscope offers four independent operable STM units which can access the sample. The lateral positions of the STM tips above the sample are monitored by a scanning electron microscope (SEM) (Nanofocus by Staib). N-type silicon substrates in (111) and (100) surface orientation were chosen with resistivities of $2-10 \Omega \mathrm{m}$. Their surfaces were cleaned $e x$ situ by etching with hydrofluoric acid and passivating the surface with hydrogen. Afterwards, the Si samples were further prepared by several cycles of flash annealing up to $1500 \mathrm{~K}$ under UHV conditions followed by cooling to room temperature. This ensures a clean and flat surface onto which a thin layer (equivalent to about 3-6 nm) of bismuth was evaporated from a home-built e-beam evaporator. During deposition the Si samples were kept at low temperature $(130 \mathrm{~K})$. After deposition the Bi films were annealed to slightly above room temperature (400 $\mathrm{K})$. This procedure results in thin, closed (111) oriented layers of bismuth with high interface and surface quality. ${ }^{[33-36]}$ This can easily be checked by low energy electron diffraction (LEED) and STM (Fig. 1a-d). Using a shadow mask, several areas with thin Bi films are prepared simultaneously on the same sample. Both the $\mathrm{Si}$ spots (marked by blue circles) as well as 


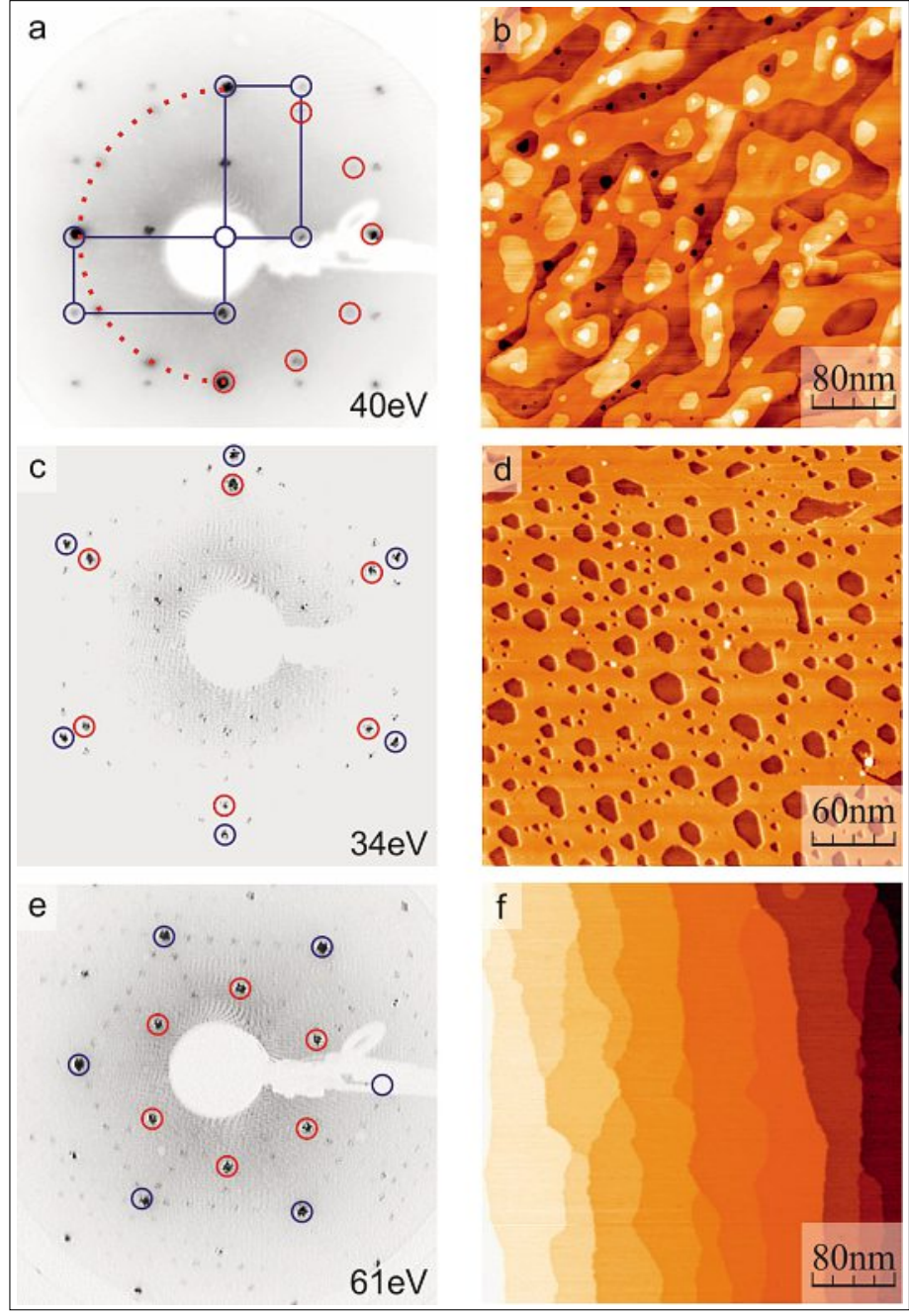

Fig. 1. (a) LEED and corresponding STM data (b) of a $3 \mathrm{~nm}$ thick $\mathrm{Bi}(111)$ film on Si(100)-2×1. Tunneling parameters $I_{t}=20$ $\mathrm{pA}$ and $\mathrm{V}_{\text {bias }}=-1.5 \mathrm{~V}$. Due to two Bi(111) domains rotated by $90^{\circ}$, the LEED shows 12 spots. The z-scale of the STM image shows a corrugation of about four Bi bilayers (c and d). Corresponding data for the $\mathrm{Bi}(111)$ film prepared on a $\mathrm{Si}(111)-7 \times 7$ substrate. $\left(I_{t}=80 \mathrm{pA}\right.$ and $V_{\text {bias }}$ $=1.8 \mathrm{~V}$ ). The surface roughness in the STM data corresponds to one Bi bilayer (e and f). Data for the $\mathrm{Si}(111)$ $(\sqrt{3} \times \sqrt{3})$-Ag surface. Several $(\sqrt{3} \times \sqrt{3})$ terraces are observed. (I $=10 \mathrm{pA}$ and $\mathrm{V}_{\text {bias }}=2$ $\mathrm{mV})$. The blue circles in the LEED data indicate substrate spots of $\mathrm{Si}(111)$ and $\mathrm{Si}(100)$ while the red circles mark $\mathrm{Bi}(111)$ spots (a and $b$ ) or spots of the $(\sqrt{ } 3 \times \sqrt{ } 3)-A g$ surface reconstruction respectively (e). the $\mathrm{Bi}(111)$ spots (marked by red circles) can be imaged using LEED (Fig. 1a,c). On the $\mathrm{Si}(100)$ substrate two $\mathrm{Bi}(111)$ domains rotated by $90^{\circ}$ are present on the surface, therefore the LEED images (Fig. 1a) exhibit $12 \mathrm{Bi}(111)$ spots. $\mathrm{Bi}(111)$ grows in a bilayered structure and the films show a low corrugation of some (one to four) bilayers of bismuth. Depending on the exact amount of bismuth during growth, either ad-islands of truncated triagonal shape (Fig. 1b) or bilayer deep holes (Fig. 1d) are observed in the STM images and may vary along the bismuth film. The BEEM experiments were carried out at a sample temperature of $150 \mathrm{~K}$. The $\operatorname{Bi}(111)$ surface also serves as a template for organic molecules. We deposited sub-monolayer coverages of PTCDA and $\mathrm{C}_{60}$ onto the flat $\mathrm{Bi}(111)$ surface at room temperature by sublimation from a Knudsen cell.

In the case of the potentiometry measurements beside a $\mathrm{Bi}(111)$ film as described above, the silver induced $\sqrt{3} \times \sqrt{3}$ reconstructed $\mathrm{Si}(111)$ surface was chosen.[37] Here, after flash annealing the Si sample to $1500 \mathrm{~K}$, the sample was kept at $800 \mathrm{~K}$ while the surface was exposed to a flux of silver. Afterwards the sample was cooled down to room temperature. This results in the $\operatorname{Si}(111)(\sqrt{3} \times \sqrt{3})-\mathrm{Ag}$ surface (Fig. 1e and $\mathrm{f}$ ) while the additional amount of silver aggregates into (111)-oriented silver islands. A miscut of $0.5^{\circ}$ results in a stepped surface exhibiting about 30 steps/ $\mu \mathrm{m}$ as observed in the STM images (Fig. 1f). The potentiometry measurements were carried out at room temperature.

All image acquisition was done using the open source software $\mathrm{GSxM}^{[38]}$ and data processing was done using WxSM. ${ }^{[39]}$

\section{Ballistic Electron Emission Microscopy}

Ballistic electron emission microscopy (BEEM), introduced by Bell and Kaiser, ${ }^{[20,21]}$ allows the characterization of ballistic charge carrier transport through a Schottky device by analyzing the fraction of ballistic transmitted charge carriers. Thus, scattering processes (i.e. energy loss events) at injection, propagation and transmission become accessible. Several review articles provide an overview on this technique. ${ }^{40-43]}$

Fig. 2 shows a schematic of the BEEM experiment. Hot electrons are injected by the tunneling tip either directly into the bismuth film or via an organic adsorbate. While the tunneling bias at the tip $\left(\mathrm{V}_{\text {tip }}\right)$ determines the energy distribution, the momentum distribution of the injected electrons is determined by the geometry of the tunneling junction and the involved electronic states. In our experimental setup a second tip of the individually controllable STMs is also brought into tunneling above the Bi film and gently pushed into the surface to provide an Ohmic contact to the metal layer. Thus, the potential of the $\mathrm{Bi}$ film can be defined separately. The backside of the Si substrate is highly doped so that an Ohmic back contact is ensured. As a result the initial total tunneling current can be separated into two circuits. The charge carriers losing energy by interacting with the Bi film are collected by the tip which contacts the $\mathrm{Bi}(111)$ film. The charges propagating ballistically through the metal film may reach the Schottky barrier at the metal/semiconductor interface and enter the $\mathrm{Si}$ substrate if their energy is sufficient. The resulting 'ballistic' current is collected at the semiconductor and will be referred to as the BEEM current $\left(\mathrm{I}_{\text {ballistic }}\right)$ in the following. If the BEEM current is detected as a function of the lateral position of the STM tip at a constant bias voltage, a BEEM image showing the transmission of ballistic

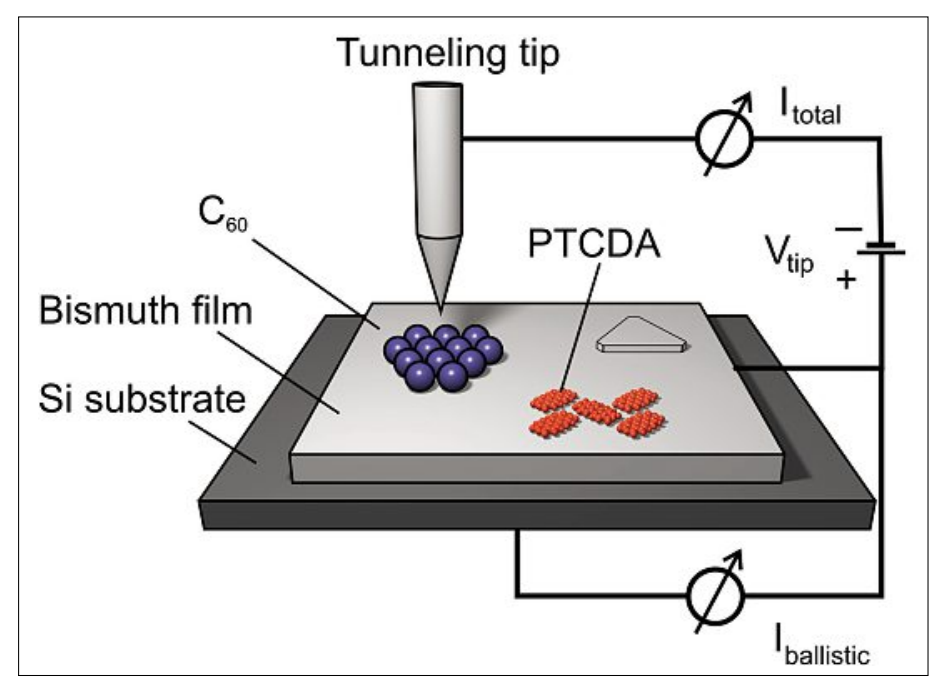

Fig. 2. Schematic of the BEEM setup. A tip of an STM injects hot electrons $\left(I_{\text {total }}\right)$ into a metal layer, either directly or via an organic adsorbate. Since both the metal layer as well as the silicon substrate are contacted, the ballistic fraction in the tunneling current $\left(I_{\text {bellisid }}\right)$ can be measured individually. Adapted with permission from A. Bannani et al., Science 2007, 315 , 1824. 


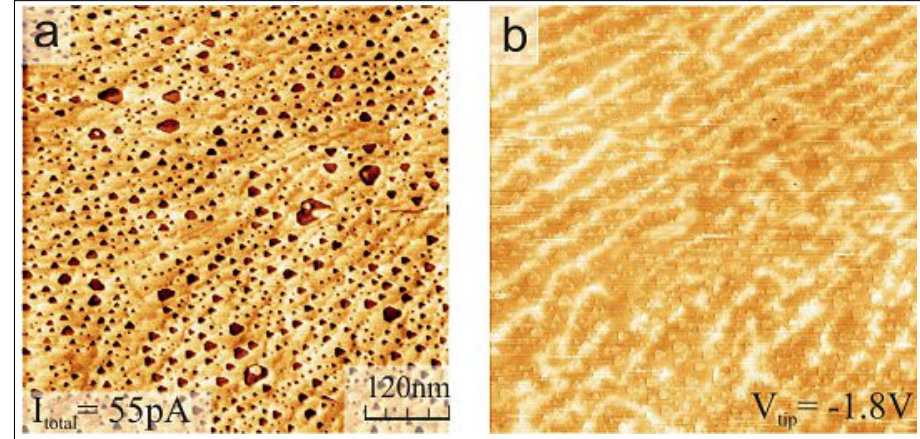

Fig. 3. (a) Topography image of $\mathrm{Bi}(111)$ on $\mathrm{Si}(100)$ exhibiting triangularshaped holes of one Bi bilayer depth. The steps of the Si substrate are slightly visible through the Bi(111) film. (b) The corresponding BEEM image shows a long-range wave-like pattern. The BEEM current varies from $3 \mathrm{pA}$ to $6 \mathrm{pA}$ (dark to bright). $\left(\mathrm{I}_{\mathrm{t}}=55 \mathrm{pA}\right.$ and $\left.\mathrm{V}_{\text {tip }}=-1.8 \mathrm{~V}\right)$

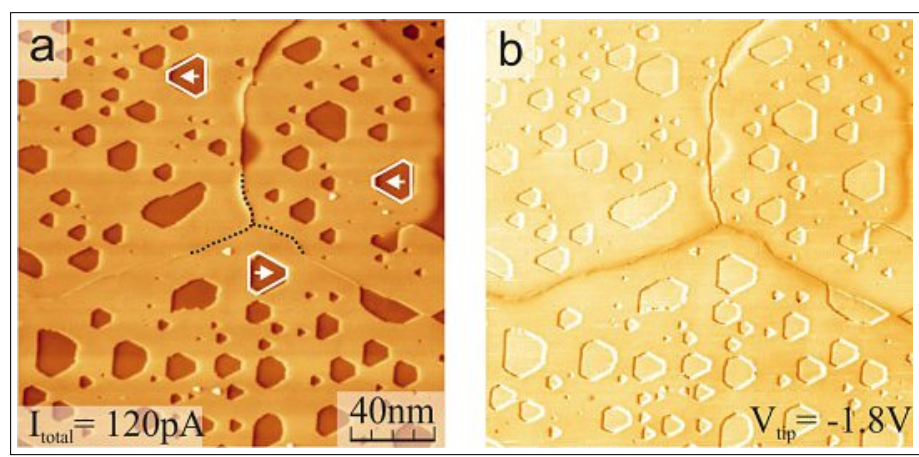

Fig. 4. (a) Topography and (b) corresponding BEEM images for $\mathrm{Bi}(111)$ on $\mathrm{Si}(111)$. The overall BEEM current varies from $0 \mathrm{pA}$ to $12 \mathrm{pA}$. Three $\mathrm{Bi}(111)$ domains are imaged, indicated by the orientation of the truncated triangular-shaped holes (see arrows in a). Three faint lines in the topography correspond to dark appearing lines in the BEEM image. In addition, at each step edge of the topography a strong BEEM signal (b) is visible. $\left(\mathrm{I}_{\mathrm{t}}=120 \mathrm{pA}\right.$ and $\left.\mathrm{V}_{\text {tip }}=-1.8 \mathrm{~V}\right)$ electrons can be acquired simultaneously to the topography. On the other hand, at a fixed lateral position ballistic electron emission spectroscopy (BEES) can be performed by varying the applied bias voltage at constant tunneling current or at constant tunneling distance. Both result in energy resolved spectra. Since the outcome is only $1 \%$ to $10 \%$ of the initial total tunneling current, $\mathrm{I}_{\text {ballistic }}$ is measured using a current to voltage amplifier with a gain of $10^{10} \mathrm{~V} / \mathrm{A}$ and a bandwidth up to $300 \mathrm{~Hz}^{[44]}$ while the overall tunneling current can be measured using a current to voltage amplifier with a gain of $10^{9} \mathrm{~V} / \mathrm{A}$. If the silicon substrate is n-doped, the emerging Schottky barrier acts as a barrier for electrons.

\subsection{Bi(111) on Si(100) and Si(111)}

Fig. 3 summarizes the findings for BEEM measurements on $\mathrm{Bi}(111)$ films prepared on a $\mathrm{Si}(100)-2 \times 1$ substrate.

The topography (a) exhibits a low surface corrugation with some holes in form of triangles or truncated triangles of one bilayer depth. However, the small corrugation of the underlying $\mathrm{Si}$ steps is still visible through the metal film. The corresponding BEEM image (b) (bright is equal to higher BEEM current) shows a long range wave-like pattern whose periodicity seems to correspond to the underlying stepped Si surface structure. In addition, a faint BEEM contrast at step edges of the $\mathrm{Bi}(111)$ film is visible. As checked by scanning in forward and backward scan directions, the step edge contrast in the case of $\mathrm{Bi} / \mathrm{Si}(100)$ is only due to a small electronic crosstalk of the total tunneling current signal (see also ref. [24]), while the wave-like contrast is well reproduced. The onset of the BEEM current determined by ballistic electron emission spectroscopy (BEES) is found at $(0.58 \pm 0.04) \mathrm{eV}$ for the system $\mathrm{Bi}(111)$ on $\mathrm{Si}(100)$. $^{\text {[24] }}$

The corresponding data set for $\mathrm{Bi}(111)$ on $\mathrm{Si}(111)-7 \times 7$ is shown in Fig. 4. The overview scan (a) exhibits a very similar topography of the $\mathrm{Bi}(111)$ surface as compared to $\mathrm{Bi}(111) / \mathrm{Si}(100)$ while the BEEM contrast (b) differs significantly. Here, the BEEM signal is strongly increased at the step edges to a value up to $12 \%$ of the total tunneling current as compared to flat surface areas exhibiting $7 \%$ of the total tunneling current. In this case the check for electronic crosstalk and scan-related artifacts reveals that the major contribution to the step edge signal in the BEEM current is a true signal. ${ }^{[24]}$ Nevertheless, a faint scan artifact comparable to $\mathrm{Bi}(111) / \mathrm{Si}(100)$ is also present in the scan. However, its contribution can be evaluated from the data set and is found to be negligible. Three dark, pronounced lines are found in the BEEM signals which correspond to domain boundaries of the $\mathrm{Bi}(111)$ film. These domain boundaries are found as a faint line in the topography image (indicated by black dotted lines in Fig. 4a). As determined by BEES the onset of the BEEM current in the case $\mathrm{Bi}(111) / \mathrm{Si}(111)$ has a value of $(0.68 \pm 0.03)$ eV. ${ }^{[24]}$

\subsection{BEEM on Organic Molecules}

Fig. 5 shows the topography and BEEM data for the organic adsorbates $\mathrm{C}_{60}$ and PTCDA simultaneously adsorbed onto the $\mathrm{Bi}(111)$ surface. The $\mathrm{Si}$ substrate is (100)-oriented. The $\mathrm{C}_{60}$ molecules aggregate in closed packed islands with a triangular shape similar to the $\mathrm{Bi}(111)$ islands. The packing of the $\mathrm{C}_{60}$ molecules has a similar in-plane lattice parameter as a bulk fcc(111) $\mathrm{C}_{60}$ crystal.[45] The PTCDA molecules arrange in the well-known herringbone structure ${ }^{[27]}$ covering part of the $\mathrm{Bi}(111)$ surface. Depending on the quality of the STM tip, the herringbone structure may not be resolved in the topography (Fig. 5a). Nevertheless, the BEEM images show
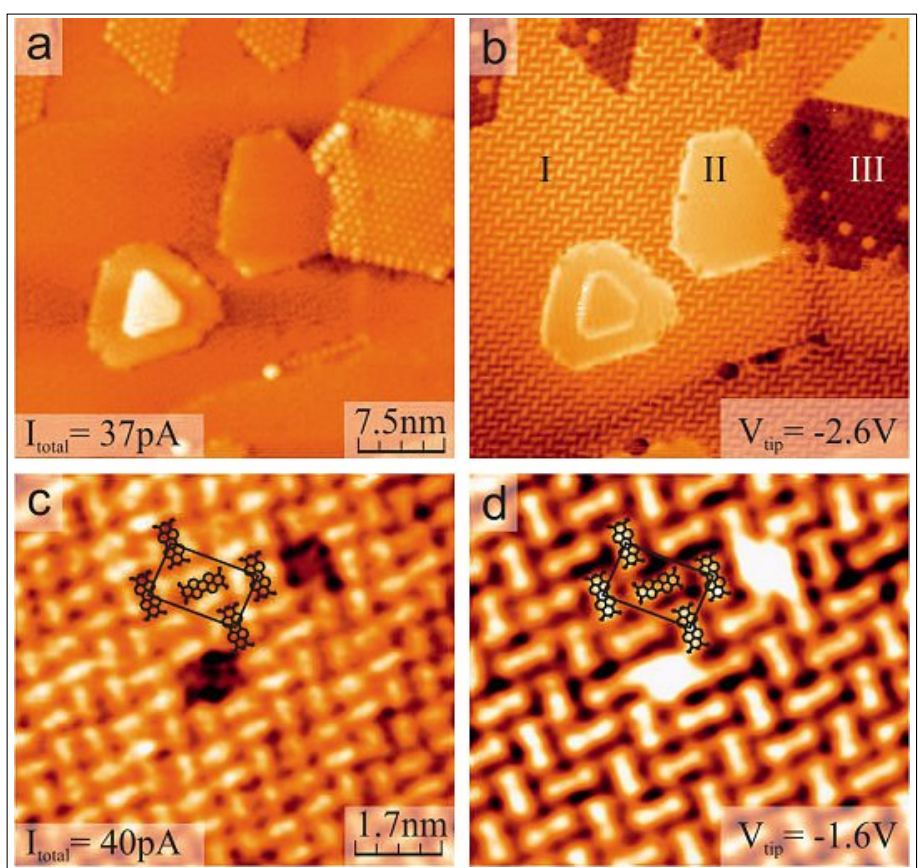

Fig. 5. (a) Topography and corresponding BEEM image (b) of the $\mathrm{Bi}(111)$ surface under the co-adsorption of PTCDA and $\mathrm{C}_{60}$ molecules. The BEEM current varies from 0.2 $\mathrm{pA}$ to $5 \mathrm{pA}$. $\left(\mathrm{I}_{\text {total }}=37\right.$ $\mathrm{pA}$ and $\mathrm{V}_{\text {tip }}=-2.5 \mathrm{~V}$ ). (c) High-resolution STM image and (d) corresponding BEEM image of a PTCDA domain including the PTCDA unit cell. A Gaussian filter was applied to (c) and (d). Two missing PTCDA molecules are observed in the topography, thus the BEEM current above those positions corresponds to the bare $\mathrm{Bi}(111)$ surface. $\left(I_{\text {total }}=\right.$ $40 \mathrm{pA}$ and $\mathrm{V}_{\text {tip }}=-1.6 \mathrm{~V}$ ) 


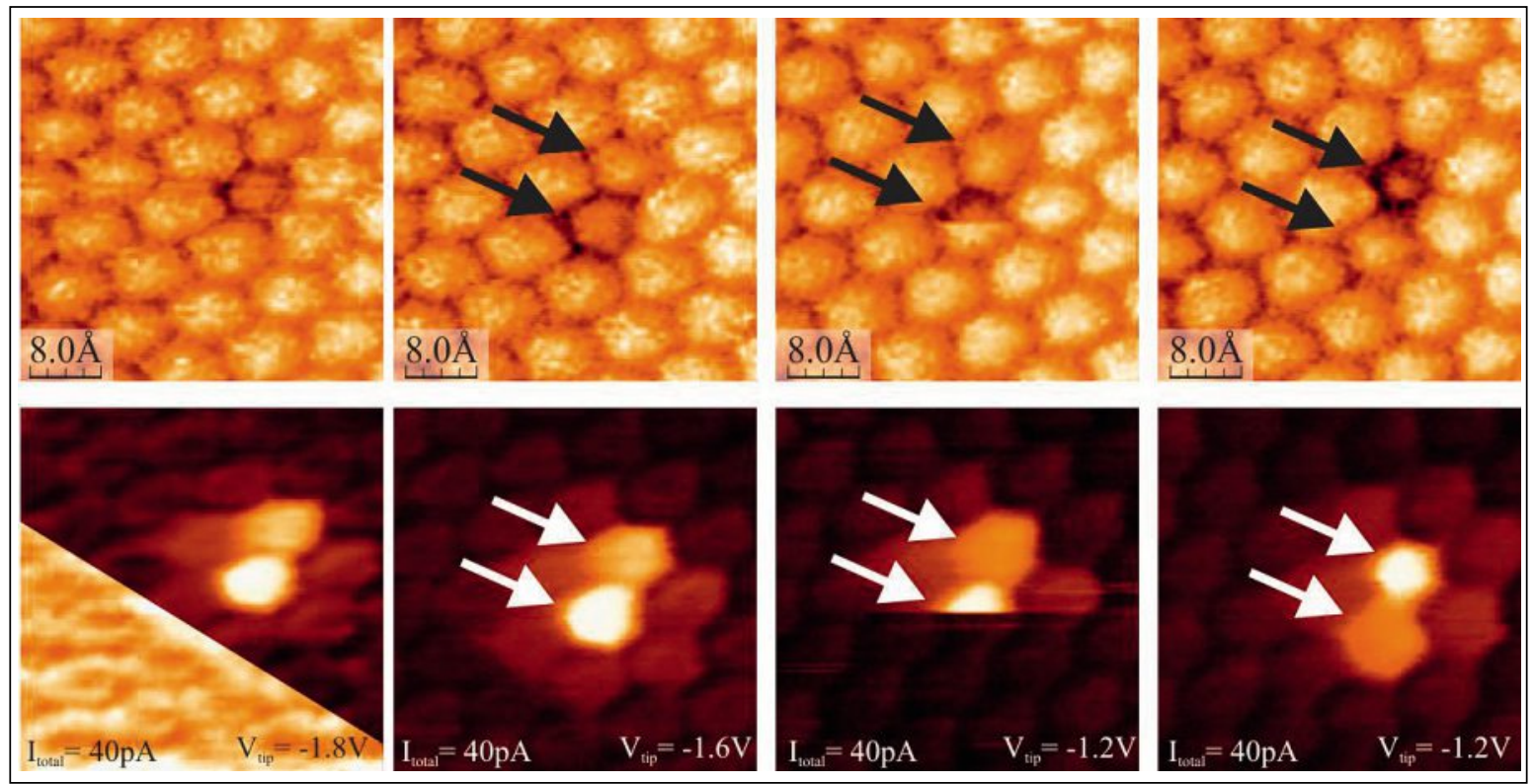

Fig. 6. Upper row: Topogaphy images above a $C_{60}$ island at varying bias voltages between -1.8 to $-1.2 \mathrm{~V}$ at a tunneling current of $40 \mathrm{pA}$. Lower row: corresponding BEEM images. The high bias voltage image at $-1.8 \mathrm{~V}$ (lefthand side) exhibits an internal structure, resulting in a donut-like shape. The color table of the intersected part within the left BEEM image was shifted to enhance the contrast. At lower bias voltage the internal structure vanishes. Upon subsequent scanning the bright appearance of the $\mathrm{C}_{60}$ hops to a neighboring molecule. The BEEM current within the images varies between $0.4 \mathrm{pA}$ and 6 pA depending on the tunneling voltage. Adapted with permission from A. Bannani et al., Science 2007, 315, 1824.

a very pronounced contrast for both molecular species (Fig. 5b). Especially above the PTCDA islands, the contrast variation in the BEEM signal is rather strong varying by about $4 \mathrm{pA}$ corresponding to a relative modulation of more than $50 \%$ of the BEEM current. The contrast variation in the BEEM image can be distinguished into three different regions corresponding to PTCDA islands (I), the bare $\mathrm{Bi}(111)$ surface (II) and $\mathrm{C}_{60}$ islands (III) (see Fig. 5b).

Above both molecular species the BEEM current is attenuated in comparison to the bare $\mathrm{Bi}(111)$ surface (Fig. 5b) for the accessible range of electron energies between $0 \mathrm{eV}$ to $3 \mathrm{eV}$. Nevertheless, in both cases the periodicity of the molecular arrangements is resembled in the BEEM contrast. We want to note that in many cases the BEEM contrast can be used to distinguish between molecular domains and the bare $\mathrm{Bi}(111)$ surface even though in conventional topographic imaging the tip resolution may be poor (compare Fig. $5 \mathrm{a}$ and $\mathrm{b}$ ). A high-resolution BEEM image of the PTCDA domain (Fig. 5d) shows that the molecules appear as elongated protrusions, which are aligned to the perylene body of the molecule. However, above the anhydride groups, the BEEM signal is slightly stronger resulting in a bone-like appearance. The BEEM appearance of the molecules remains the same for various energies (not shown here) and differs from the topographic appearance (Fig. 5c). In the topography the PTCDA molecules appear as two bright protrusions which are separated by a darker line in the middle.
For the $\mathrm{C}_{60}$ molecules the BEEM contrast depends on the applied tunneling voltage, i.e. electron energy.

Up to an energy of $1.6 \mathrm{eV}$ the $\mathrm{C}_{60}$ appear to be homogeneously transmitting (Fig. 6), while above this energy they exhibit a donut-like shape, with a darker less ballistic transmitting part in the middle (Fig. 6 , left). It was carefully checked that the contrast variation of this donut-like shape is found inside the $\mathrm{C}_{60}$ molecules and not in-between the molecules. For the range of energy the BEEM image also reproduces the molecular arrangement as seen in the topography. ${ }^{[32]}$ One specific molecule appears to be somewhat lower in the topography, exhibiting a much higher BEEM contrast. Upon scanning at low voltage, this effect may hop to a neighboring molecule as shown in Fig. 6 (left to right). The appearance in the topography image as well as the high BEEM transmission is transferred to the next molecule. The effect is prominent for two $\mathrm{C}_{60}$ molecules while the adjacent molecules are slightly affected resulting in a halo like appearance.

\subsection{Discussion of the BEEM Data}

The different onset of the BEEM current in the case of a $\mathrm{Si}(111)$ substrate as compared to $\mathrm{Si}(100)$ originates from the different matching between the bandstructures of the $\mathrm{Bi}(111)$ film and the corresponding Si substrate. ${ }^{[24]}$

For $\mathrm{Bi}(111)$ on $\mathrm{Si}(111)$, electron injection near the $\Gamma$ point is not possible. Even though injection near the $\Gamma$ point into the $\mathrm{Bi}(111)$ film is possible there are no matching states at the interface to the Si(111) substrate (Fig. 7a and c). At higher lateral momentum the $\mathrm{Si}(111)$ substrate offers states e.g. near the M point, but at low energies, i.e. $0.58 \mathrm{eV}$, there is no overlap to states in the $\operatorname{Bi}(111)$ film. However, at higher energy, i.e. $0.67 \mathrm{eV}$, there is some overlap between states in the $\mathrm{Bi}(111)$ film and the $\operatorname{Si}(111)$ substrate near the $M$ point. Thus, the onset of the BEEM current is not only determined by the Schottky barrier but also by the overlap of the electronic bandstructure at the interface. ${ }^{[24]}$ The energy needed to enter the semiconductor can be written as $E=E_{\|}+E_{\iota}$, while $E_{\perp}$ is determined by the Schottky barrier and $E_{\|}$is the additional energy needed to increase the bandstructure overlap. As a result the BEEM current onset is delayed to $(0.68 \pm 0.03) \mathrm{eV}$.

In contrast the $\mathrm{Si}(100)$ substrate allows injection near $\Gamma$ (Fig. $7 b$ and d) so that no additional lateral electron momentum (i.e. no additional energy contribution $E_{\|}$) is needed to enter the semiconductor. The onset of the BEEM current $(0.58 \pm 0.04)$ $\mathrm{eV}$ is not delayed. The BEEM data reveal the band matching between the Bi layer and the Si substrate for different Si surface orientations.

This matching of the bandstructures at the $\mathrm{Bi} / \mathrm{Si}$ interface is visualized by BEEM images acquired for both $\mathrm{Si}$ substrates. For the Si(111) substrate at a given energy above the BEEM current onset, the overall BEEM current on the $\mathrm{Bi}$ terraces does not vary significantly unless the injection takes place near a Bi step edge (Fig. 4b). Upon 


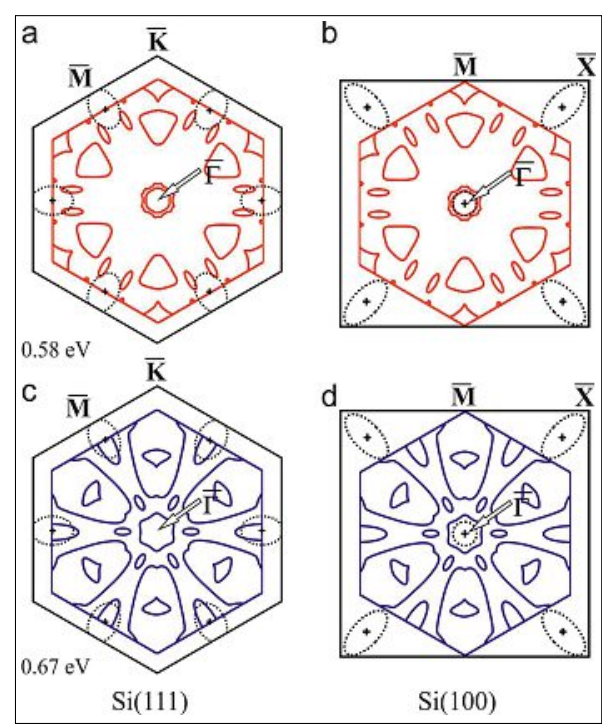

Fig. 7. Projected electronic states of the $\mathrm{Si}(111)$ (a, c) and the $\mathrm{Si}(100)$ (b, d) substrates (black). The projected electronic states of the $\mathrm{Bi}(111)$ thin film at $0.58 \mathrm{eV}$ (red) and $0.67 \mathrm{eV}$ (blue) are superimposed. Reproduced with permission from C. A. Bobisch et al., Phys. Rev. Letts. 2009, 102, 136807. Copyright 2009, American Physical Society.

injection near a step edge an increased percentage of electrons carry a higher lateral electron momentum $\mathrm{k}_{\|}$when reaching the interface to the silicon substrate. These electrons can contribute to the BEEM current so that the BEEM signal near step edges is increased.

The situation is different for a $\mathrm{Si}(100)$ $2 \times 1$ substrate. Injection near $\Gamma$ of the $\mathrm{Si}(100)$ substrate is possible, so that no additional $\mathrm{k}_{\|}$is necessary to enter the semiconductor. Therefore, the surface step edges do not show an effect in the BEEM signal. There is a BEEM current variation corresponding to the buried $\mathrm{Si}$ steps. This variation is attributed to the conservation of the $\mathrm{Si}(100)-2 \times 1$ reconstruction underneath the $\mathrm{Bi}$ film. Although the $\mathrm{Bi}$ film contains several domains, these usually grow across several $\mathrm{Si}$ terraces. This is indicated by domain boundaries crossing several Si steps (see Fig. 3). ${ }^{[25]}$ For each neighboring terrace the orientation of the $2 \times 1$ reconstruction of the $\operatorname{Si}(100)$ surface is rotated by $90^{\circ}$, resulting in two different interfaces to the $\mathrm{Bi}(111)$ film so that the injection process into adjacent $\mathrm{Si}$ terraces may differ.

In order to understand the BEEM data for $\mathrm{C}_{60}$ or PTCDA molecules deposited onto the $\mathrm{Bi}$ surface, the transmission properties related to the $\mathrm{Bi}(111) / \mathrm{Si}$ sample have to be known. The adsorption geometry of PTCDA varies on different metals, as the bonding distances between the molecules' atoms and the surface atoms differ. On a silver substrate the corner oxygen atoms are somewhat closer to the surface than the perylene body, suggesting a stronger coupling. ${ }^{[46]}$ The PTCDA BEEM data suggest a similar behavior for PTCDA on $\mathrm{Bi}(111)$. An increased BEEM current at the short sides of the PTCDA molecule is an indication for a more effective coupling or a reduced distance to the $\mathrm{Bi}(111)$ surface. Since the electrons from the LUMO states are blocked by the Schottky barrier of the $\mathrm{Bi} / \mathrm{Si}$ diode, the overall appearance in the BEEM image can be attributed to the LUMO+1 state of the molecule.[47] This is further corroborated by BEES data acquired above the PTCDA domains (not shown here). In addition to an attenuated overall outcome of the BEEM current, the form of the spectra differs from the spectra on the bare $\mathrm{Bi}(111)$ surface. This behavior can be well fitted by introducing a second barrier to the fit function at a higher energy above the original BEEM onset. The extracted value of the second barrier $(1.0 \pm 0.1 \mathrm{eV})$ could be explained by introducing the PTCDA molecule into the tunneling pathway such that the electrons can enter the LUMO+1 state at this energy (see supporting online material in ref. [32]). The appearance of the topographic images corresponds to the LUMO state of the PTCDA molecule. This is no contradiction since for the topographic images all injected electrons can contribute which may be dominated by the LUMO, while the BEEM image is filtered in energy by the Schottky barrier.

A similar behavior is found if the BEEM data for $\mathrm{C}_{60}$ molecules are analyzed. The appearance of the $\mathrm{C}_{60}$ molecules in the BEEM images above $1.6 \mathrm{eV}$ can be related to the LUMO+1 state of the $\mathrm{C}_{60}$. The electrons originating from the LUMO state are blocked at the Schottky interface. Since the sample is only cooled down to $150 \mathrm{~K}$, the $\mathrm{C}_{60}$ may still undergo a rotational motion within the islands, inducing the ring-like BEEM contrast. For $\mathrm{C}_{60}$ molecules current transport favorably occurs along the $\mathrm{C}-\mathrm{C}$ bonds. ${ }^{[48]}$ Therefore, a higher scattering probability can be assumed if the injecting STM tip is placed above the center of the molecule rather than near the outer rim of each molecule. A higher fraction of ballistic electrons near the rim of the molecules will enter the Bi surface resulting in the donut-like shaped BEEM images. The particularly bright appearing $\mathrm{C}_{60}$ molecule within the island almost shows the same transmission as the bare Bi substrate. This may be attributed to a doping by $e . g$. an adatom on top or between two molecules. ${ }^{[49]}$ This is supported by the fact that the bright appearance occurs for at least two $\mathrm{C}_{60}$ close to each other. Upon subsequent scanning the effect may hop between both affected molecules, as demonstrated in Fig. 6.

\section{Scanning Tunneling Potentiometry}

The lateral variation of the electrochemical potential $\mu_{\mathrm{ec}}$ can be studied using the technique of scanning tunneling potentiometry (STP), introduced by Muralt and Pohl in 1986. ${ }^{[22,23]}$ So far only a limited number of publications are available, demonstrating the capability to study the electrochemical potential with atomic resolution. ${ }^{[37,50,51]}$ A schematic of the experimental setup is shown in Fig. 8.

We use two tips of the multiprobe STM to establish Ohmic contacts to the surface. Thus by applying a voltage between both contact tips a potential gradient along a surface structure between the contacts can be created resulting in a current through the surface. A third STM tip measures the topography between the contact tips. Simultaneously, the electrochemical potential is mapped by realizing a wheatstone-bridge like setup. A small alternating voltage is superimposed, such that the overall tunneling current $\mathrm{I}_{\text {tunnel }}$ consists of two contributions, a DC component and an AC component. By controlling the distance between the STM tip and the sample as a function of the $\mathrm{AC}$ component of the tunneling current $\mathrm{I}_{\mathrm{AC}}$, the topography can be obtained in an ordinary STM image at the Fermi energy. A second feedback loop controls the overall DC voltage between the tunneling tip and the sample in such a way that the net DC tunneling current $I_{D C}$ becomes zero. Thereby, the voltage at the tip and the sample correspond to each other, i.e. the output voltage of the second feedback loop measures the electrochemical potential at each lateral tip position. Since this is done during the tunneling experiment, both the potential as well as the topography can be measured simultaneously with atomic resolution. Care was taken that the feedback loop for the potential is about one order of magnitude slower than the $\mathrm{z}$ feedback loop, which ensures that both feedback loops do not couple to each other. The experimental setup can be further improved by introducing a fourth STM tip which provides a reference potential in close vicinity to the measuring STP tip. This reduces the influence of fluctuations in the pathways between the two contacting STM tips which are further away from the studied surface area. The experiments described here were performed at room temperature and a base pressure better than $5 \times 10^{-10}$ mbar. ${ }^{[51]}$

\subsection{Bi(111)}

We prepared two rather large $\mathrm{Bi}$ covered areas which are connected by a narrow Bi stripe using a shadow mask. ${ }^{[51]}$ The large $\mathrm{Bi}$ areas are used to establish contacts with two tips of the four-probe- 


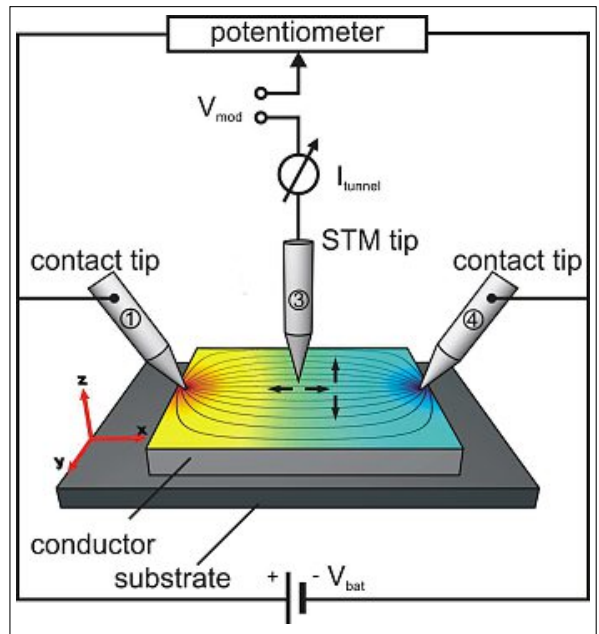

Fig. 8. Schematic to measure the electrochemical potential under a STM tip. Two contact tips establish an Ohmic contact to a surface, thus a current can be applied. The resulting potential distribution along the surface as well as the surface morphology is mapped by the third STM tip. A small AC voltage $\left(\mathrm{V}_{\text {mod }}\right)$ applied to the tunneling tip allows the topography to be measured by using the corresponding $A C$ component of the tunneling current $\left(\mathrm{I}_{\mathrm{AC}}\right)$.

STM setup (see SEM image in Fig. 9a). The two pad geometry allows a high current density to be achieved in the narrow $\mathrm{Bi}$ stripe. The third STM tip maps the topography and the electrochemical potential of the Bi(111) surface in the STP mode. If current fluctuations near the contacting tips occur the fourth tip may be set in contact to the Bi stripe close to the STP tip providing a reference potential. Then, the STP tip images the electrochemical potential in reference to the fourth tip. However, usu- ally this was not necessary and a three-tip geometry was sufficient.

Fig. 9b shows the topography above the $\mathrm{Bi}$ stripe and the corresponding potential maps for both lateral current polarities. In the topography (Fig. 9b) the stepped surface corrugation of the Si substrate is still visible under the Bi layer. Domain boundaries are also visible as faint lines in the topography (marked by the arrows in Fig. $9 b)$. The potential maps acquired in STP mode show an overall potential drop of 500 $\mu \mathrm{V}$ within the observed area. This potential decay is inverted if the lateral current is inverted (c and d). A close inspection of the domain boundary (arrows in Fig. 9b) reveals that the potential transition is less smooth at this position. ${ }^{[51]} \mathrm{A}$ faint step-like behavior may be attributed. However, there is also some finestructure visible within the $\mathrm{Bi}(111)$ domains whose sign does not invert upon current reversal.

\subsection{Si(111) $(\sqrt{3} \times \sqrt{3})-\mathrm{Ag}$}

During the preparation of the $\mathrm{Si}(111)$ $(\sqrt{3} \times \sqrt{3})$-Ag surface the additional amount of silver aggregates into Ag islands (Fig. $10 a, b, c)$. These islands serve as ideal contact pads so that we use them to establish Ohmic contacts by gently pushing two of our STM tips into them (Fig. 10b and Fig. $10 \mathrm{c})$. In order to vary the direction of the current with respect to the surface step edge directions, a surface area exhibiting four Ag islands in close vicinity was chosen. The red bars in Fig. 9a indicate the orientation of the surface steps.

Fig. 10d shows a large overview scan between the contact tips. The stepped surface structure originates from the miscut of the Si wafer and consists mostly of mono

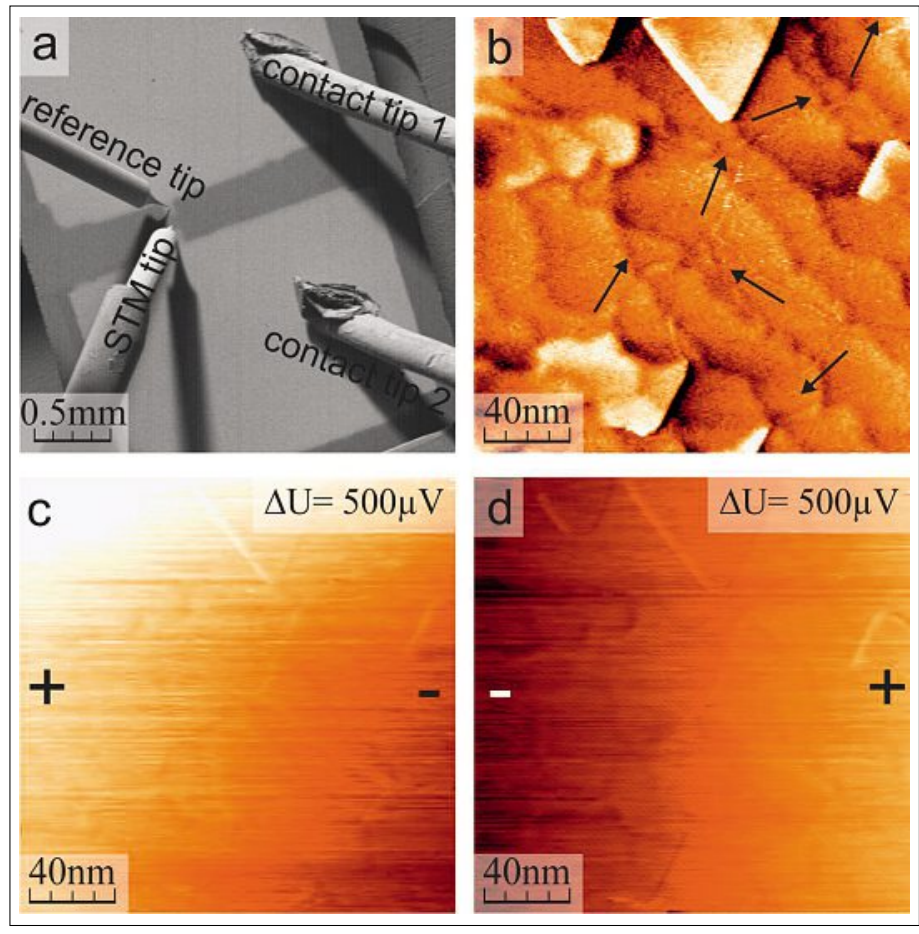

Fig. 9. (a) SEM overview image, showing two STM tips contacting the large Bi(111) pads. A third tip measures the topography and the potential on the $\mathrm{Bi}(111)$ stripe between the contact pads. A forth tip may be optionally used as a reference electrode. The topography (b) exhibits the underlying Si steps which are overgrown by the Bi film. The arrows mark $\mathrm{Bi}$ domain boundaries. (c) and (d) show the corresponding potential map inverted applied voltages between the contact tips. In both cases the potential varies by about 500 $\mu \mathrm{V}$ within the image. and double atomic steps. On the reconstructed terraces a few one-layer-thick Ag islands are found as well.

To study the influence of the surface morphology on the potential variation, two experimental geometries were chosen in which the current is applied either parallel or perpendicular to the surface steps. This is realized by placing the contact tips accordingly to the different contact positions as depicted on the SEM images in Fig. 10b and $\mathrm{c}$.

For both geometries the topography and the electrochemical potential were measured for the same area. Figs 10d to $f$ summarize the findings for both cases. The topography in (d) can be compared to the potential images (e and f) which show a different contrast. It is obvious that the potential varies rather abruptly at surface step edges and stays more or less constant on the terraces. Step-like changes of the potential also occur at domain boundaries of the surface reconstruction. If the current is applied perpendicular to the surface steps, the major potential variation happens at the step edges (d). In contrary, the contribution of domain boundaries in the reconstructed surface structure which are perpendicular to the surface steps edges is mainly visible if the current direction is parallel to the step edges (f). It should be noted, that there exist two different type of domain boundaries within the surface reconstruction, both of which exhibit different potential drops. Also a faint gradient of the electrochemical potential is visible on the terraces. The current density within the obtained image area is evaluated to $0.4 \mathrm{~A} / \mathrm{m}$ for the current perpendicular to the step edges and $2 \mathrm{~A} / \mathrm{m}$ if the current is applied parallel to the surface steps. ${ }^{[37]}$ Using simulations based on a simple Ohmic network, we extracted values for the specific resistivities of single steps, multiple steps and domain boundaries and the 2DEG itself. ${ }^{[37]}$ As can be seen in Table 1 , the values for both current directions are different, e.g. in the case of a single step by about one order of magnitude.

\subsection{Discussion}

Bulk Bi offers a low conductivity but metallic surface states are present in the Bi(111) surface which can contribute to the surface conductivity. ${ }^{[52,53]}$ In the STP data the $\mathrm{Bi}(111)$ surface mainly exhibits a potential gradient along the whole surface area independent of surface roughness, i.e. surface steps. This may be attributed to electron-phonon scattering. In some cases a rather abrupt variation of the potential may be attributed to a domain boundary of the $\operatorname{Bi}(111)$ film. The charge transport can be either governed by metallic surface states or the bulk states of the Bi(111) film. For a $\mathrm{Bi}(111)$ single crystal there is no significant contribution of the metallic sur- 


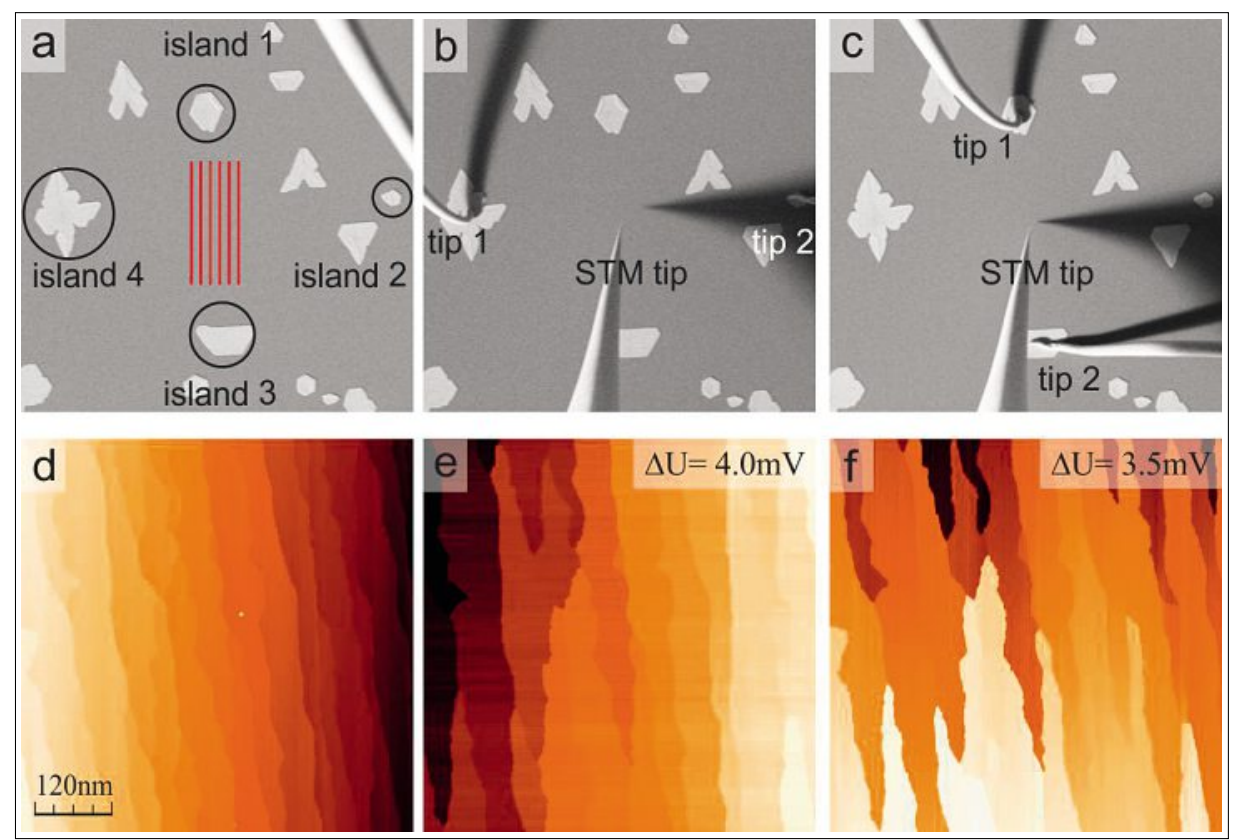

Fig. 10. (a-c) SEM images of the tips during the scanning tunneling potentiometry measurement on the $\mathrm{Si}(111)-(\sqrt{3} \times \sqrt{3})-\mathrm{Ag}$ surface. The red lines in (a) indicate the surface step directions. Islands 1 to 4 (circles in (a)) are used as contact pads. A voltage is applied between tip 1 and tip 2 either perpendicular to the surface steps (b) or parallel to the surface steps (c). (d) STM topography measured between tip 1 and 2. (e) Potential map of the surface corresponding to the geometry in (b). (f) Potential map corresponding to the geometry in (c).

Table 1. Adapted with permission from J. Homoth et al. Nano Lett. 2009, 9, 1588-1592). Copyright (2009) American Chemical Society.

\begin{tabular}{|l|l|l|}
\hline type of defect & $\begin{array}{l}\text { current perpendicular } \\
\text { to the steps }\end{array}$ & $\begin{array}{l}\text { current parallel to the } \\
\text { steps }\end{array}$ \\
\hline $\begin{array}{l}\sigma_{2 \mathrm{DEG}}, 10^{-6} \Omega^{-1} / \square \\
\text { single step, } 10^{-6} \Omega^{-1} \mathrm{~m}^{-1}\end{array}$ & $>2000$ & $270 \pm 70$ \\
double step, $10^{-6} \Omega^{-1} \mathrm{~m}^{-1}$ & $3200 \pm 500$ & $<300$ \\
domain boundary $\mathrm{A}, 10^{-6} \Omega^{-1} \mathrm{~m}^{-1}$ & $700 \pm 300$ & $<40$ \\
domain boundary B, $10^{-6} \Omega^{-1} \mathrm{~m}^{-1}$ & $>10,000$ & $15 \pm 5$ \\
\hline
\end{tabular}

face states to the conductivity reported. ${ }^{[54]}$ Assuming transport through the bulk, in our experiment a specific conductivity of $(1.24 \pm 0.05) \times 10^{6} \Omega^{-1} \mathrm{~m}^{-1}$ can be evaluated ${ }^{[51]}$ which is higher than the literature value for bulk Bi of $0.77 \times 10^{6} \Omega^{-1} \mathrm{~m}^{-1}$. For thin $\mathrm{Bi}(111)$ films grown on $\mathrm{Si}$ substrates an increased resistivity due to surface roughening by adsorbing additional $\mathrm{Bi}$ atoms is discussed. ${ }^{[52]}$ The local density of states near the Fermi level of the Bi(111) surface is highly influenced by defects such as adsorbates. ${ }^{[53]}$ However, our data show almost no impact of surface defects to the electrochemical potential. The potentiometry features near step edges can be attributed to a small thermovoltage which is superimposed to the potentiometry signal. Upon current flow through the bismuth film, the surface may be heated leading to a small temperature difference between the Bi(111) surface and the STM tip. This creates a thermovoltage which is measured by the voltage feedback loop during the STP measurement as well. ${ }^{[55-57]}$ These ef- fects are easily distinguished from effects related to the electronic transport since they are not related to the current direction. However, the features at the domain boundaries are inverted upon current reversal, i.e. they are attributed to a decrease of the conductivity.

Hirahara et al. ${ }^{[58]}$ reported a major contribution from the metallic surface states to the conductivity for thin highly crystalline Bi films. They determine the conductivities of the surface state $\left(\sigma_{\mathrm{ss}}\right.$ $\left.=1.5 \times 10^{-3} \Omega^{-1} / \square\right)$ and a $2 \mathrm{D}$ conductiv$\square)$. Taking the thickness of our film (3-5 $\mathrm{nm})$ into account the expected conductivity as derived from their experiment would be $\sigma_{\text {total }}=\sigma_{\text {ss }}+\sigma_{\text {film }}=\left(1.5 \times 10^{-3} \Omega^{-1} / \square\right.$ $\left.+3.6 \times 10^{-3} \Omega^{-1} / \square\right)=(5.1 \pm 1.0) \times 10^{-3} \Omega^{-1} / \square$. This fits quite well to our value of $\sigma_{\text {stp }}=$ (5.02 \pm 0.66$) \times 10^{-3} \Omega^{-1} / \square$ if we determine the 2D conductivity of our film from the STP data. Therefore both the surface states as well as the thin film contribute to the observed conductivity. ity of one bilayer $\left(\sigma_{\text {bilayer }}=3.4 \times 10^{-4} \Omega^{-1}\right.$ /
The situation is different for the $\operatorname{Si}(111)-\sqrt{ } 3 \times \sqrt{3}$ :Ag surface. The majority of charge carriers is mediated by the surface states which are well decoupled from the bulk. ${ }^{[37]}$ Electrons in these surface states show a parabolic dispersion and thus can be described as a free two-dimensional electron gas in the surface. The major contribution to the overall current flux is mediated through these states. The values for the specific conductivities result from the incident angle of the electrons impinging on the surface defects. This incident angle depends on the overall contact geometry, thus if the macroscopic current direction is more or less perpendicular to the steps then the microscopic angle of the electrons will also be perpendicular. The variation of the specific conductivities for the different directions (Table 1) can be explained by assuming that the electrons are transmitted through a tunneling barrier at the step edge. A rectangular-shaped barrier already allows simulation of the barrier data quite well. ${ }^{[37]}$ The exact shape of the barrier is not important. Assuming plane waves, the transmission can be evaluated analytically leading to maximal transmission for an incident angle of $90^{\circ}$ and less than $1 \%$ for an angle greater than $70 \%$. Thus, the observed resistivities are a result of the transmission probability at the step edge. This is also true for multiple steps or domain boundaries.

\section{Conclusion}

We analyzed electron transport phenomena at interfaces and surfaces using two three-terminal STM techniques, ballistic electron emission microscopy (BEEM) and scanning tunneling potentiometry (STP)

The transport of hot electrons through $\mathrm{Bi}(111)$ films and the organic adsorbates PTCDA and $\mathrm{C}_{60}$ is studied using the BEEM technique. This gives insight into the transport mechanism perpendicular to the surface plane into a metal film. The data reveals that the overlap of the bandstructures of both the silicon substrate and the metal film play an important role for the transmission of ballistic electrons. Due to the conservation of lateral electron momentum at the metal/semiconductor interface, the BEEM contrast for $\mathrm{Bi}(111)$ surface steps differs significantly for the $\mathrm{Si}(111)$ and the Si(100) substrate. Thus, the initial energy and momentum distribution at the metal/ vacuum interface plays an important role for the ballistic electron transmission.

This injection dependency can be used to study transport through organic adsorbates. The BEEM contrast above organic adsorbates is determined by the coupling of the adsorbate to the $\operatorname{Bi}(111)$ surface. 
BEEM images of $\mathrm{C}_{60}$ and PTCDA covered areas exhibit sub-molecular lateral resolution so that pathways inside the molecules are visualized. The appearance in the BEEM images is closely related to the available electronic states of the molecules. Thus, the coupling between the molecular states and the $\mathrm{Bi}(111)$ becomes accessible.

In scanning tunneling potentiometry, the $\operatorname{Bi}(111)$ film mainly exhibits electron phonon scattering, manifesting a monotonous gradient of the electrochemical potential. The applied current is mediated through both the $\mathrm{Bi}$ film and the $\mathrm{Bi}(111)$ surface states. In contrast, the two-dimensional electron gas system in the surface states of the silver induced $\mathrm{Si}(111)$ $(\sqrt{3} \times \sqrt{3})$-Ag surface shows a small variation of the electrochemical potential on flat surface terraces, but a strong step-like variation of the electrochemical potential at surface defects, i.e. domain boundaries and surface steps. The macroscopic observed resistivity of such a surface is given by the sum over various elementary contributions like a step like decay and a small potential gradient on the reconstructed terraces.

Both techniques, BEEM and STP, give access to scattering processes of charge carriers with atomic precision and are applicable for devices with a buried energy barrier or for two-dimensional electron gas systems at surfaces.

\section{Acknowledgements}

We thank the German Research Council (DFG) for their support through the Sonderforschungsbereich (SFB) 616. We gratefully acknowledge fruitful and stimulating discussions with A. Lorke and $\mathrm{H}$. Nienhaus. This work could not have been accomplished without the help and contributions of G. Bihlmayer (Institut für Festkörperforschung and Institute for Advanced Simulation, Forschingszentrum Jülich), J. Homoth and M. Wenderoth (4. Physikalisches Institut, University of Göttingen) and our former co-workers A. Bannani, M. Matena, E. Zubkov. All of these were highly involved in the experiments and the interpretation of the data. Additional thanks go to A. M. Bernhart and M R. Kaspers for their contributions to the experiments. We also thank D. Utzat for designing and constructing our STM-related electronics.

Received: August 5, 2011
[1] G. Binnig, H. Rohrer, C. Gerber, E. Weibel, Appl. Phys. Lett. 1982, 40, 178.

[2] G. Binnig, H. Rohrer, C. Gerber, E. Weibel, Phys. Rev. Lett. 1983, 50, 120.

[3] G. Binnig, H. Rohrer, C. Gerber, E. Weibel, Surf. Sci. 1983, 131, L379.

[4] R. M. Feenstra, A. P. Fein, Phys. Rev. B 1985 32, 1394.

[5] C. Wöll, S. Chiang, R. J. Wilson, P. H. Lippel, Phys. Rev. B 1989, 39, 7988

[6] J. Viernow, D. Y. Petrovykh, A. Kirakosian, J.-L. Lin, F. K. Men, M. Henzler, F. J. Himpsel, Phys. Rev. B 1999, 59, 10356.

[7] M. Kulawik, N. Nilius, H.-P. Rust, H.-J. Freund, Phys. Rev. Lett. 2003, 25601.

[8] R. Fasel, M. Parschau, K.-H. Ernst, Angew. Chem. Int. Ed. 2003, 41, 5178 .

[9] B. C. Stipe, M. A. Rezaei, W. Ho, Science 1998 279, 1907.

[10] M. F. Crommie, C. P. Lutz, D. M. Eigler, Nature 1993, 363, 524

[11] S. Hasegawa, P. Avouris, Phys. Rev. Lett. 1993, $71,7,1071$

[12] J. I. Pascual, J. Gómez-Herrero, C. Rogero, A. M. Baró, D. Sánchez-Portal, E. Artacho, P. Ordejón, J. M. Soler, Chem. Phys. Lett. 2000, $321,78$.

[13] B. C. Stipe, M. A. Rezaei, W. Ho, Science 1998 , 280, 1732 .

[14] J. I. Pascual, J. J. Jackiw, Z. Song, P. S. Weiss, H. Conrad, H.-P. Rust, Phys. Rev. Lett. 2001, 86, 1050.

[15] S. Heinze, M. Bode, A. Kubetzka, O. Pietzsch, X. Nie, S. Blügel, R. Wiesendanger, Science 2000, 288, 1805.

[16] M. Bode, E. Y. Vedmedenko, K. von Bergmann, A. Kubetzka, P. Ferriani, S. Heinze, R Wiesendanger, Nature Materials 2006, 5, 477.

[17] X. H. Qiu, G. V. Nazin, W. Ho, Science 2003, 299, 542.

[18] C. Chen, C. A. Bobisch, W. Ho, Science 2009, $325,981$.

[19] C. Chen, P. Chu, C. A. Bobisch, D. L. Mills, W Ho, Phys. Rev. Lett. 2010, 105, 217402.

[20] W. J. Kaiser, L. D. Bell, Phys. Rev. Lett. 1988 60, 1406.

[21] L. D. Bell, W. J. Kaiser, Phys. Rev. Lett. 1988, 61, 2368.

[22] P. Muralt, D. W. Pohl, Appl. Phys. Lett. 1986, $48,8$.

[23] P. Muralt, H. Meier, D. W. Pohl, H. W. M Salemink, Appl. Phys. Lett. 1987, 50, 1352.

[24] C. A. Bobisch, A. Bannani, Y. M. Koroteev, G. Bihlmayer, E. V. Chulkov, R. Möller, Phys. Rev. Lett. 2009, 102, 136807.

[25] A. Bannani, C. A. Bobisch, R. Möller, Appl. Phys. Lett. 2008, 93, 032111.

[26] R. Yamachika, M. Grobis, A. Wachowiak, M. F. Crommie, Science 2004, 304, 281

[27] F. S. Tautz, Prog. Surf. Sci. 2007, 82, 479.

[28] C. Weiss, C. Wagner, C. Kleimann, M. Rohlfing, F. S. Tautz, R. Temirov, Phys. Rev. Lett. 2010 105, 086103 .

[29] C. Troadec, K. E. J. Goh, JVST 2010, 28, C5F1.

[30] J. C. W. Song, K. E. J. Goh, N. Chandrasekhar, C. Troadec, Phys. Rev. B 2009, 79, 165313.

[31] C. A. Bobisch, A. Bannani, A. Bernhart, E Zubkov, B. Weyers, R. Möller, J. Phys.: Conf. Ser. 2008, 100, 052064.
[32] A. Bannani, C. Bobisch, R. Möller, Science 2007, 315, 1824

[33] T. Nagao, J. T. Sadowski, M. Saito, S. Yaginuma, Y. Fujikawa, T. Kogure, T. Ohno, Y. Hasegawa, S. Hasegawa, T. Sakurai, Phys. Rev. Lett. 2004 $93,105501$.

[34] G. Jnawali, H. Hattab, B. Krenzer, M. Horn-von Hoegen, Phys. Rev. B 2006, 74, 195340.

[35] C. Bobisch, A. Bannani, M. Matena, R. Möller, Nanotechnology 2007, 18, 055606.

[36] H. Hattab, E. Zubkov, A. Bernhart, G. Jnawali, C. Bobisch, B. Krenzer, M. Acet, R. Möller, M Horn-von-Hoegen, Thin Solid Films 2008, 516, 8227.

[37] J. Homoth, M. Wenderoth, T. Druga, L. Winking, R. G. Ulbrich, C. A. Bobisch, B. Weyers, A. Bannani, E. Zubkov, A. M. Bernhart, M. R. Kaspers, R. Möller, Nano Letters 2009, 9 , 1588.

[38] P. Zahl, M. Bierkandt, S. Schröder, A. Klust, Rev. Sci. Instrum. 2003, 74, 1222.

[39] I. Horca, R. Fernandez, J. M. GomezRodriguez, J. Colchero, J. Gomez-Herrero, A. M. Baro, Rev. Sci. Instrum. 2007, 78, 013705.

[40] M. Prietsch, Phys. Rep. 1995, 253, 163.

[41] L. D. Bell, W. J. Kaiser, Annu. Rev. Mater. Sci. 1996, 26, 189.

[42] P. L. de Andres, F. J. Garcia-Vidal, K. Reuter, F. Flores, Prog. Surf. Sci. 2001, 66, 3.

[43] W. Yi, A. J. Stollenwerk, V. Narayanamurti, Surf. Sci. Rep. 2009, 64, 169 .

[44] Design and assembly by D. Utzat.

[45 J. T. Sadowski, R. Z. Bakhtizin, A. I. Oreshkin, T. Nishihara, A. Al-Mahboob, Y. Fujikawa, K. Nakajima, T. Sakurai, Surf. Sci. 2007, 601, L136.

[46] S. Duhm, A. Gerlach, I. Salzmann, B. Bröker, R. L. Johnson, F. Schreiber, N. Koch, Org. El. 2008, 9,111

[47] R. Temirov, S. Soubatch, A. Luican, F. S. Tautz, Nature 2006, 444, 350

[48] T. Ono, K. Hirose, Phys. Rev. Lett. 2007, 98, 026804.

[49] G. V. Nazin, X. H. Qiu, W. Ho, J. Chem. Phys. Comm. 2005, 122, 181105-1-4.

[50] B. G. Briner, R. M. Feenstra, T. P. Chin, J. M. Woodall, Phys. Rev B 1996, 54, 8 .

[51] A. Bannani, C. A. Bobisch, R. Möller, Rev. Sci. Instrum., 2008 79, 083704.

[52] G. Jnawali, T. Wagner, H. Hattab, R. Möller, A. Lorke, M. Horn-von Hoegen, Surf. Sci. Nanotechnol. 2010, 8, 27.

[53] M. C. Cottin, C. A. Bobisch, J. Schaffert, G Jnawali, A. Sonntag, G. Bihlmayer, R. Möller, Appl. Phys. Lett. 2011, 98, 022108.

[54] J. W. Wells, K. Handrup, J. F. Kallehauge, L. Gammelgaard, P. Boggild, M. B. Balslev, J. E. Hansen, P. R. E. Petersen, P. Hofmann, J. Appl. Phys. 2008, 104, 053717 .

[55] D. Hoffmann, A. Rettenberger, C. Baur, K. Läuger, J. Y. Grand, R. Möller, App. Phys. Lett. 1995, 67, 1217.

[56] D. Hoffmann, A. Rettenberger, K. Läuger, J. Y. Grand, R. Möller, J. Vac. Sci. Technol. A 1997, $15,1418$.

[57] D. Hoffmann, A. Rettenberger, K. Läuger, J. Y. Grand, R. Möller, Phys. Rev. B 1995, 52, 13796.

[58] T. Hirahara, I. Matsuda, S. Yamazaki, N. Miyata, T. Nagao, S. Hasegawa, Appl. Phys. Lett. 2007, 91, 202106. 